\title{
Exponential Convergence Bounds using Integral Quadratic Constraints
}

\author{
Ross Boczar Laurent Lessard Benjamin Recht
}

\begin{abstract}
The theory of integral quadratic constraints (IQCs) allows verification of stability and gain-bound properties of systems containing nonlinear or uncertain elements. Gain bounds often imply exponential stability, but it can be challenging to compute useful numerical bounds on the exponential decay rate. In this work, we present a modification of the classical IQC results of Megretski and Rantzer [13] that leads to a tractable computational procedure for finding exponential rate certificates. We demonstrate the effectiveness of our method via a numerical example.
\end{abstract}

\section{Introduction}

In robust control problems, we seek absolute performance guarantees about a system in the presence of bounded uncertainty. Examples of such results include the small gain theorem \& passivity theory [20], dissipativity theory [19], and integral quadratic constraints (IQCs) [13].

In this paper, we present a modification of IQC theory, the most general of the aforementioned tools, that allows one to certify exponential stability rather than just boundedinput bounded-output (BIBO) stability. Moreover, we can compute numerical bounds on the exponential decay rate of the state.

Even when BIBO stable systems are exponentially stable, the estimates of the exponential decay rates provided by standard IQC theory are typically very conservative. We will show that this conservatism can be greatly reduced if we directly certify exponential stability and use the method presented herein to compute the associated decay rate.

Our modified IQC analysis was successfully applied in [12] to analyze convergence properties of commonly-used optimization algorithms such as the gradient descent method. These algorithms converge at an exponential rate when applied to strongly convex functions, and the modified IQC analysis automatically produces very tight bounds on the convergence rates.

Another potential application is in time-critical applications such as embedded model predictive control, where it is vital to have robust guarantees that the desired error bounds will be achieved in the allotted time without overflow errors and in spite of fixed-point arithmetic. See $[8]$ and references therein.

A special case. As previously noted, exponential stability certificates are often conservative when they are derived from $L_{2}$ gain bounds. However, it is well known that exponential stability can be proven directly in some special cases. To illustrate this fact, consider a discrete linear time-invariant (LTI) plant $G$ with state-space realization $(A, B, C, D)$. Suppose $G$ is connected in feedback with a passive nonlinearity $\Delta$. A sufficient condition for BIBO stability is that there exists a positive definite matrix $P \succ 0$ 
and a scalar $\lambda \geq 0$ satisfying the linear matrix inequality (LMI)

$$
\left[\begin{array}{cc}
A & B \\
I & 0
\end{array}\right]^{\top}\left[\begin{array}{cc}
P & 0 \\
0 & -P
\end{array}\right]\left[\begin{array}{cc}
A & B \\
I & 0
\end{array}\right]+\lambda\left[\begin{array}{cc}
0 & C^{\top} \\
C & D+D^{\top}
\end{array}\right] \prec 0
$$

If we define $V(x):=x^{\top} P x$, then (1) implies that $V$ decreases along trajectories: $V\left(x_{k+1}\right) \leq V\left(x_{k}\right)$ for all $k$. BIBO stability then follows from positivity and boundedness of $V$. But observe that when (1) holds, we may replace the right-hand side by $-\varepsilon P$ for some $\varepsilon>0$ sufficiently small. We then conclude that $V\left(x_{k+1}\right) \leq(1-\varepsilon) V\left(x_{k}\right)$ for all $k$ and exponential stability follows. We can then maximize $\varepsilon$ subject to feasibility of (1) to further improve the rate bound.

Unfortunately, the simple trick shown above does not work in the general IQC setting due to the different role played by $P$ in the associated LMI. The LMI used in IQC theory comes from the Kalman-Yakubovich-Popov (KYP) lemma and although it is structurally similar to (1), $P$ is not positive definite in general and $V$ may not decrease along trajectories.

Our key insight is that with a suitable modification to both the LMI and the IQC definition, we obtain a condition that can certify exponential stability.

The paper is organized as follows. We cover some related work in the remainder of the introduction, we explain our notation and some basic results in Section 2, we develop and present our main result in Section 3, and we discuss computational considerations in Section 4. Finally, we present an illustrative example demonstrating the usefulness of our result in Section 5, and we make some concluding remarks in Section 6.

Related work. It is noted in $[13,15]$ that BIBO stability often implies exponential stability. In particular, we get exponential stability if the nonlinearity satisfies an additional fading memory property. The proof of this result is chiefly concerned with showing existence of an exponential decay rate. Although the proof constructs an exponential rate, the construction is based on the assumed $L_{2}$ gain of the linear map, and thus can be very conservative.

Other proofs of exponential stability have appeared in the literature for specific classes of nonlinearities. Some examples include $[4,11]$, which treat sector-bounded nonlinearities, and [9], which treats nonlinearities satisfying a Popov IQC. These works exploit LMI modifications akin to the one shown with (1) earlier in this section.

The sequel is inspired by the recent paper [12], which presents an approach for proving the robust exponential stability of optimization algorithms. The approach of [12] uses a time-domain formulation of IQCs modified to handle exponential stability. In contrast, the present work develops the aforementioned exponential stability modification entirely in the frequency domain and clarifies its connection to the seminal IQC results in [13].

\section{Notation and preliminaries}

We adopt a setup analogous to the one used in [13], with the exception that we will work in discrete time rather than continuous time. The conjugate transpose of a vector $v \in \mathbb{C}^{n}$ is denoted $v^{*}$. The unit circle in the complex plane is denoted $\mathbb{T}:=\{z \in \mathbb{C}|| z \mid=1\}$ The $z$-transform of a time-domain signal $x:=\left(x_{0}, x_{1}, \ldots\right)$ is denoted $\hat{x}(z)$ and defined as $\hat{x}(z):=\sum_{k=0}^{\infty} x_{k} z^{-k}$.

A Hermitian positive definite (semidefinite) matrix $M$ is denoted $M \succ 0(M \succeq 0)$. Function composition is denoted $(g \circ f)(x):=g(f(x))$. A sequence $u=\left(u_{0}, u_{1}, \ldots\right)$ is said to be in $\ell_{2}$ if $\sum_{k=0}^{\infty}\left|u_{k}\right|^{2}<\infty$. A sequence $u_{k}$ is said to be in $\ell_{2}^{\rho}$ for some $\rho \in(0,1)$ 
if the sequence $\left(\rho^{-k} u_{k}\right)$ is in $\ell_{2}$, i.e. $\sum_{k=0}^{\infty} \rho^{-2 k}\left|u_{k}\right|^{2}<\infty$. Note that $\ell_{2}^{\rho} \subset \ell_{2}$. Let $\mathcal{R} \mathcal{H}_{\infty}^{m \times n}$ be the set of $m \times n$ matrices whose elements are proper rational functions with real coefficients analytic on the closed unit disk.

Consider the standard setup of Fig. 1. The block $G$ contains the known LTI part of the system while $\Delta$ contains the part that is uncertain, unknown, nonlinear, or otherwise troublesome. The interconnection is said to be well-posed if the map $(v, w) \mapsto(e, f)$ has a

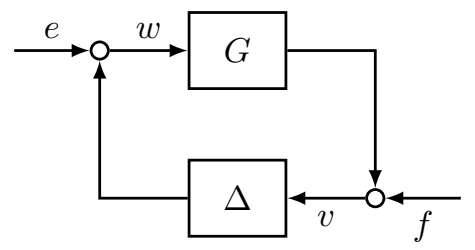

Figure 1: Linear time-invariant system $G$ in feedback with a nonlinearity $\Delta$.

causal inverse. The interconnection is said to be BIBO stable if, in addition, there exists some $\gamma>0$ such that when $G$ is initialized with zero state,

$$
\|v\|^{2}+\|w\|^{2} \leq \gamma\left(\|e\|^{2}+\|f\|^{2}\right)
$$

for all square-summable inputs $f$ and $e$, and where $\|\cdot\|$ denotes the $\ell_{2}$ norm. Finally, the interconnection is exponentially stable if there exists some $\rho \in(0,1)$ and $c>0$ such that if $f=0$ and $e=0$, the state $x_{k}$ of $G$ will decay exponentially with rate $\rho$. That is,

$$
\left\|x_{k}\right\| \leq c \rho^{k}\left\|x_{0}\right\| \quad \text { for all } k .
$$

We now present the classical IQC definition and stability result, which will be modified in the sequel to guarantee exponential convergence. These results are the discrete-time analog of the main IQC results of Megretski and Rantzer [13].

Definition 1 (IQC). Signals $y \in \ell_{2}$ and $u \in \ell_{2}$ with associated $z$-transforms $\hat{y}(z)$ and $\hat{u}(z)$ satisfy the IQC defined by a Hermitian complex-valued function $\Pi$ if

$$
\int_{\mathbb{T}}\left[\begin{array}{l}
\hat{y}(z) \\
\hat{u}(z)
\end{array}\right]^{*} \Pi(z)\left[\begin{array}{l}
\hat{y}(z) \\
\hat{u}(z)
\end{array}\right] d z \geq 0 .
$$

$A$ bounded operator $\Delta$ satisfies the IQC defined by $\Pi$ if (2) holds for all $y \in \ell_{2}$ with $u=\Delta(y)$. We also define $\mathrm{IQC}(\Pi(z))$ to be the set of all $\Delta$ that satisfy the IQC defined by $\Pi$.

Theorem 2 (Stability result). Let $G(z) \in \mathcal{R H}_{\infty}^{m \times n}$ and let $\Delta$ be a bounded causal operator. Suppose that:

i) for every $\tau \in[0,1]$, the interconnection of $G$ and $\tau \Delta$ is well-posed.

ii) for every $\tau \in[0,1]$, we have $\tau \Delta \in \operatorname{IQC}(\Pi(z))$.

iii) there exists $\varepsilon>0$ such that

$$
\left[\begin{array}{c}
G(z) \\
I
\end{array}\right]^{*} \Pi(z)\left[\begin{array}{c}
G(z) \\
I
\end{array}\right] \preceq-\varepsilon I, \quad \forall z \in \mathbb{T} .
$$

Then, the feedback interconnection of $G$ and $\Delta$ is stable. 


\section{Frequency-domain condition}

In this section, we augment Definition 1 and the classical result of Theorem 2 to derive a frequency-domain condition that certifies exponential stability.

Definition 3. The operators $\rho_{+}, \rho_{-}$are defined as the time-domain, time-dependent multipliers $\rho^{k}, \rho^{-k}$, respectively, where $\rho \in(0,1)$ is a defined constant.

Remark 4. The operator $\rho_{-} \circ\left(G(z) \circ \rho_{+}\right)$is equivalent to the operator $G(\rho z)$. This follows from the fact that, for any constant $a>0$ and signal $u_{k}$, the $z$-transform of $a^{-k} u_{k}$ is given by $\hat{u}(a z)$. See Fig. 2 for an illustration.

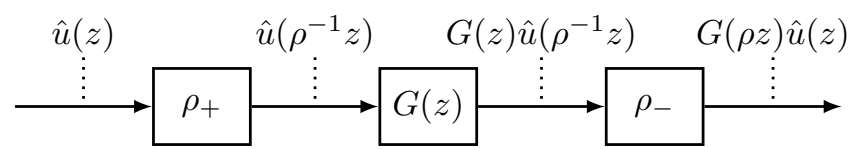

Figure 2: Illustration of Remark 4.

In order to show exponential stability of the system in Fig. 1, we will relate it to BIBO stability of the modified system shown in Fig. 3. This equivalence is closely related to the theory of stability multipliers [16].

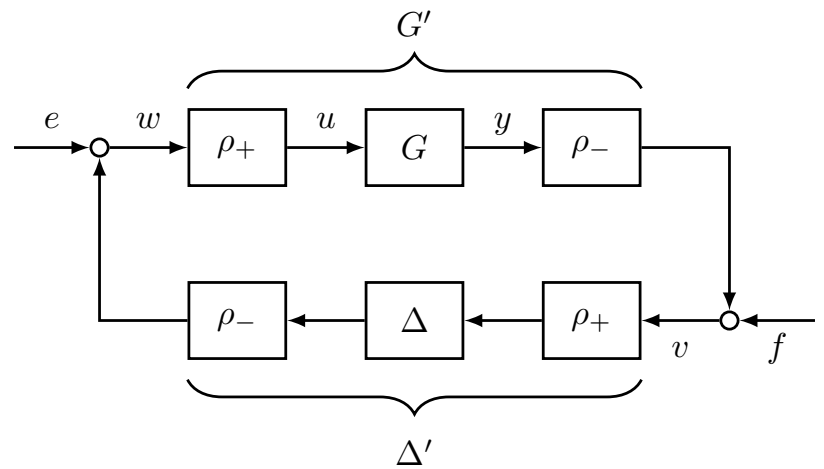

Figure 3: Modified feedback diagram with additional multipliers and inputs. For appropriately chosen $e$ and $f$ and with zero initial condition, we show how this diagram is equivalent to that of Fig. 1.

Proposition 5. Suppose $G(z)$ has a minimal realization $(A, B, C, D)$. If the interconnection in Fig. 3 is stable with zero initial condition, then the interconnection in Fig. 1 with initial state $x_{0}$ is exponentially stable.

Proof. Intuitively, if $v$ and $w$ are small in the BIBO sense compared to $e$ and $f$, then $y$ must be even smaller. A complete proof is included in the appendix.

Ideally, we would like to find a suitable redefinition of the IQC for this transformed system shown in Fig. 3. To this end, we introduce the concept of the $\rho-I Q C$.

Definition 6 ( $\rho$-IQC). Signals $y \in \ell_{2}^{\rho}$ and $u \in \ell_{2}^{\rho}$ with associated $z$-transforms $\hat{y}(z)$ and $\hat{u}(z)$ satisfy the $\rho$-IQC defined by a Hermitian complex-valued function $\Pi$ if

$$
\int_{\mathbb{T}}\left[\begin{array}{l}
\hat{y}(\rho z) \\
\hat{u}(\rho z)
\end{array}\right]^{*} \Pi(\rho z)\left[\begin{array}{l}
\hat{y}(\rho z) \\
\hat{u}(\rho z)
\end{array}\right] d z \geq 0 .
$$


$A$ bounded operator $\Delta$ satisfies the $\rho$-IQC defined by $\Pi$ if (3) holds for all $y \in \ell_{2}^{\rho}$ with $u=\Delta(y)$. We also define $\operatorname{IQC}(\Pi(z), \rho)$ to be the set of all $\Delta$ that satisfy the $\rho$-IQC defined by $\Pi$.

Note that the concept of a $\rho$-IQC generalizes that of a regular IQC. Indeed, we have $\operatorname{IQC}(\Pi(z), 1)=\operatorname{IQC}(\Pi(z))$. The restriction of $u \in \ell_{2}^{\rho}$ and $y \in \ell_{2}^{\rho}$ corresponds to the restriction of $u \in \ell_{2}$ and $y \in \ell_{2}$ in the classical definition of IQC [13]. Now equipped with $\rho$-IQCs, we can relate $\Delta^{\prime}$ in Fig. 3 to $\Delta$ in Fig. 1.

Proposition 7. Let $\Delta$ be a nonlinearity, and let $\Pi$ be a Hermitian complex-valued function. As in Fig. 3, define $\Delta^{\prime}:=\rho_{-} \circ\left(\Delta \circ \rho_{+}\right)$. Then the following statements are equivalent.

(i) $\Delta \in \operatorname{IQC}(\Pi(z), \rho)$

(ii) $\Delta^{\prime} \in \operatorname{IQC}(\Pi(\rho z))$

Proof. We define the discrete Fourier transform of the input and output of $\Delta$ as $\hat{y}(z)$ and $\hat{u}(z)$, respectively. Then, from the definition of $\rho_{+}$and $\rho_{-}$, we have that $\hat{w}(z)=\hat{u}(\rho z)$ and $\hat{v}(z)=\hat{y}(\rho z)$. Substituting into the IQC definition (2), we obtain (3) as required.

Proposition 7 is illustrated in Fig. 4.

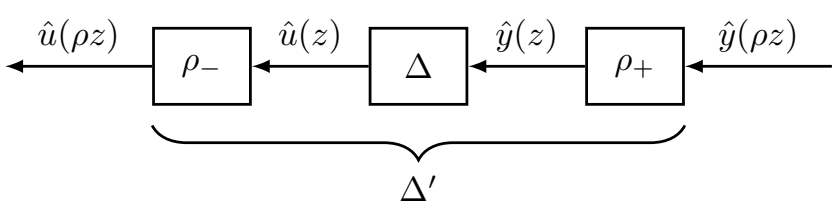

Figure 4: Illustration of Proposition 7.

We now state our main result, an exponential stability theorem analogous to the classical result in Theorem 2.

Theorem 8 (Exponential stability). Fix $\rho \in(0,1)$. Let $G(\rho z) \in \mathcal{R H}_{\infty}^{m \times n}$ and let $\Delta$ be a bounded causal operator. Suppose that:

i) for every $\tau \in[0,1]$, the interconnection of $G$ and $\tau \Delta$ is well-posed.

ii) for every $\tau \in[0,1]$, we have $\tau \Delta \in \operatorname{IQC}(\Pi(z), \rho)$.

iii) there exists $\varepsilon>0$ such that

$$
\left[\begin{array}{c}
G(\rho z) \\
I
\end{array}\right]^{*} \Pi(\rho z)\left[\begin{array}{c}
G(\rho z) \\
I
\end{array}\right] \preceq-\varepsilon I, \quad \forall z \in \mathbb{T} .
$$

Then, the interconnection of $G$ and $\Delta$ shown in Fig. 1 is exponentially stable with rate $\rho$.

Proof. Roughly, we apply Theorem 2 to the interconnection in Fig. 3 with operators $G^{\prime}$ and $\Delta^{\prime}$ and IQC $\Pi(\rho z)$.

(a) Since Fig. 1 and Fig. 3 have the same interconnection structure, well-posedness is equivalent. 
(b) Due to the equivalence of IQCs in Proposition 7,

$$
\begin{aligned}
& \tau \Delta \in \operatorname{IQC}(\Pi(z), \rho) \\
& \Longleftrightarrow \rho_{-} \circ\left((\tau \Delta) \circ \rho_{+}\right) \in \operatorname{IQC}(\Pi(\rho z)) \\
& \Longleftrightarrow \tau\left(\rho_{-} \circ\left(\Delta \circ \rho_{+}\right)\right) \in \operatorname{IQC}(\Pi(\rho z)) \\
& \Longleftrightarrow \tau \Delta^{\prime} \in \operatorname{IQC}(\Pi(\rho z)) .
\end{aligned}
$$

(c) This is condition iii) of Theorem 2 using $G^{\prime}$ and $\Delta^{\prime}$.

Thus, these three conditions ensure BIBO stability of the system in Fig. 3. We then apply Proposition 5 to arrive at exponential stability of Fig. 1.

\section{Computation}

As in the classical IQC setting, to guarantee stability, the frequency-domain inequality (FDI) (4) must be verified for every $\omega \in[0,2 \pi)$. However, if the IQC in question exhibits a factorizaton, then the discrete-time KYP Lemma can be applied to convert the infinitedimensional FDI to a finite-dimensional LMI. We now review these results.

Definition 9. We say $\Pi$ has a factorization $(\Psi, M)$ if

$$
\Pi(z)=\Psi(z)^{*} M \Psi(z),
$$

where $\Psi$ is a stable linear time-invariant system, $M$ is a constant Hermitian matrix, and $\Psi(z)^{*}$ denotes the conjugate transpose of $\Psi(z)$.

Remark 10. If $\Pi(z)$ has a factorization $(\Psi, M)$ and $\Psi(\rho z)$ is stable, then (3) is equivalent to

$$
\sum_{k=0}^{\infty} \rho^{-2 k} z_{k}^{\top} M z_{k} \geq 0, \quad \text { where } z:=\Psi\left(\begin{array}{l}
y \\
u
\end{array}\right) .
$$

This follows immediately from Parseval's theorem.

The KYP lemma, stated below, is attributed to Kalman, Yakubovich, and Popov. A simple proof and further references can be found in [14].

Lemma 11 (Discrete-time KYP Lemma). Given matrices $A, B$ and a Hermitian matrix $M$, and assuming $A$ has no eigenvalues on the unit circle, the FDI

$$
\left[\begin{array}{c}
(z I-A)^{-1} B \\
I
\end{array}\right]^{*} M\left[\begin{array}{c}
(z I-A)^{-1} B \\
I
\end{array}\right] \prec 0
$$

holds for all $z \in \mathbb{T}$ if and only if there exists a solution $P=P^{\top}$ and $\lambda \geq 0$ to the LMI

$$
\left[\begin{array}{cc}
A & B \\
I & 0
\end{array}\right]^{\top}\left[\begin{array}{cc}
P & 0 \\
0 & -P
\end{array}\right]\left[\begin{array}{cc}
A & B \\
I & 0
\end{array}\right]+\lambda M \prec 0 .
$$

Corollary 12. Suppose the realization of $G$ is given by $(A, B, C, D)$ and assume $\Pi$ has a factorization $(\Psi, M)$, where the realization of $\Psi$ is given by

$$
\Psi=\left[\begin{array}{c|cc}
A_{\Psi} & B_{\Psi_{1}} & B_{\Psi_{2}} \\
\hline C_{\Psi} & D_{\Psi_{1}} & D_{\Psi_{2}}
\end{array}\right] .
$$


Then (4) is equivalent to the existence of $P=P^{\top}$ and $\lambda \geq 0$ such that

$$
\left[\begin{array}{cc}
\hat{A}^{\top} P \hat{A}-\rho^{2} P & \hat{A}^{\top} P \hat{B} \\
\hat{B}^{\top} P \hat{A} & \hat{B}^{\top} P \hat{B}
\end{array}\right]+\lambda\left[\begin{array}{c}
\hat{C}^{\top} \\
\hat{D}^{\top}
\end{array}\right] M\left[\begin{array}{ll}
\hat{C} & \hat{D}
\end{array}\right] \prec 0
$$

where $(\hat{A}, \hat{B}, \hat{C}, \hat{D})$ are given by

$$
\left[\begin{array}{c|c}
\hat{A} & \hat{B} \\
\hline \hat{C} & \hat{D}
\end{array}\right]:=\left[\begin{array}{cc|c}
A & 0 & B \\
B_{\Psi_{1}} C & A_{\Psi} & B_{\Psi_{2}}+B_{\Psi_{1}} D \\
\hline D_{\Psi_{1}} C & C_{\Psi} & D_{\Psi_{2}}+D_{\Psi_{1}} D
\end{array}\right]
$$

Proof. A similar result is proven in [18], which we repeat here for completeness.

$$
\left[\begin{array}{c}
G(z) \\
I
\end{array}\right]^{*} \Pi(z)\left[\begin{array}{c}
G(z) \\
I
\end{array}\right]=\left[\begin{array}{l}
\star \\
\star
\end{array}\right]^{*}\left(\left[\begin{array}{l}
\hat{C}^{\top} \\
\hat{D}^{\top}
\end{array}\right] M\left[\begin{array}{ll}
\hat{C} & \hat{D}
\end{array}\right]\right)\left[\begin{array}{c}
(z I-\hat{A})^{-1} \hat{B} \\
I
\end{array}\right]
$$

where $\star$ denotes the repeated part of the quadratic form. Similarly, we have

$$
\begin{aligned}
& \rho^{-2}\left[\begin{array}{c}
G(\rho z) \\
I
\end{array}\right]^{*} \Pi(\rho z)\left[\begin{array}{c}
G(\rho z) \\
I
\end{array}\right] \\
& =\left[\begin{array}{c}
\star \\
\star
\end{array}\right]^{*}\left(\left[\begin{array}{c}
\hat{C}^{\top} \\
\hat{D}^{\top}
\end{array}\right] \rho^{-2} M\left[\begin{array}{ll}
\hat{C} & \hat{D}
\end{array}\right]\right)\left[\begin{array}{c}
(\rho z I-\hat{A})^{-1} \hat{B} \\
I
\end{array}\right] \\
& =\left[\begin{array}{c}
\star \\
\star
\end{array}\right]^{*}\left(\left[\begin{array}{l}
\hat{C}^{\top} \\
\hat{D}^{\top}
\end{array}\right] \rho^{-2} M\left[\begin{array}{ll}
\hat{C} & \hat{D}
\end{array}\right]\right)\left[\begin{array}{c}
\left(z I-\rho^{-1} \hat{A}\right)^{-1} \hat{\rho}^{-1} B \\
I
\end{array}\right]
\end{aligned}
$$

If $\rho^{-1} \hat{A}$ has no eigenvalues on the unit circle, we may then invoke Lemma 11 (applied to $\rho^{-1} \hat{A}, \rho^{-1} \hat{B}$, and the appropriate $M$ term) and multiply through by $\rho^{2}$ to show that (4) is equivalent to the existence of $P=P^{\top}$ and $\lambda \geq 0$ such that (5) holds, as required.

With the advent of fast interior-point methods to solve LMIs, the feasibility of the LMI (5) can be quickly ascertained for any fixed $\rho^{2}$. Since the size of the LMI is on the order of the size of the system $G$ and the IQC $\Pi$, most practical linear systems lead to relatively small LMIs.

Finding the best upper bound amounts to minimizing $\rho^{2}$ subject to (5) being feasible. This type of problem occurs frequently in robust control and is known as a generalized eigenvalue optimization problem (GEVP) [3]. The GEVP is not an LMI because (5) is not jointly linear in $\rho^{2}$ and $P$. One simple approach to solving the GEVP is to perform a bisection search on $\rho^{2}$, but there are more sophisticated methods available; see for example [2].

Remark 13. These results may also be carried through in continuous time. In that case, an equation analogous to (4) must be satisfied for $G(s-\lambda)$ for all $\omega \in[0, \infty)$, and can be verified by finding $P=P^{\top}, \lambda \geq 0$ such that

$$
\left[\begin{array}{cc}
\hat{A}^{\top} P+P \hat{A}-2 \lambda P & P \hat{B} \\
\hat{B}^{\top} P & 0
\end{array}\right]+\lambda\left[\begin{array}{c}
\hat{C}^{\top} \\
\hat{D}^{\top}
\end{array}\right] M\left[\begin{array}{ll}
\hat{C} & \hat{D}
\end{array}\right] \prec 0
$$

\section{$5 \quad$ Examples}

In this section, we show some classes of nonlinearities that can be described by $\rho$-IQCs and therefore used in Theorem 8 to prove robust exponential stability of an interconnected system. In the case where $\rho=1$, these $\rho$-IQCs reduce to standard IQCs [13]. This class of IQCs will be constructed for SISO systems, but they may be adapted for square MIMO systems where the nonlinearity is of the form $\operatorname{diag}(\{\Delta\})$ for a scalar $\Delta$, with little modification. 


\subsection{Pointwise IQCs}

A nonlinearity $\Delta$ satisfies a pointwise IQC with a factorization $(\Psi, M)$ if $z_{k}^{\top} M z_{k} \geq 0$ for each $k$. In other words, the IQC holds pointwise in time. In this case, $\Delta$ also satisfies the associated $\rho$-IQC for all $\rho \leq 1$. Examples of pointwise IQCs include the $\gamma$ norm-bounded $I Q C$

$$
\Pi=\left[\begin{array}{cc}
\gamma^{2} & 0 \\
0 & -1
\end{array}\right],
$$

and the $[\alpha, \beta]$ sector bounded $I Q C$, given by

$$
\Pi=\left[\begin{array}{cc}
-2 \alpha \beta & \alpha+\beta \\
\alpha+\beta & -2
\end{array}\right] .
$$

Note that the norm-bounded IQC is a special case of the sector IQC with the sector $[-\gamma, \gamma]$. These IQCs hold even if $\Delta$ is time-varying.

\subsection{Zames-Falb IQCs}

A nonlinearity $\Delta$ is slope-restricted on $[\alpha, \beta]$ where $0 \leq \alpha \leq \beta$ if the following relation holds for all $x, y$.

$$
(\Delta(x)-\Delta(y)-\alpha(x-y))^{\top}(\Delta(x)-\Delta(y)-\beta(x-y)) \leq 0 .
$$

This relation states that the chord joining input-output pairs of $\Delta$ has a slope that is bounded between $\alpha$ and $\beta$. This class of functions satisfies the so-called Zames-Falb family of IQCs $[7,21]$. We give the definition below.

Proposition 14. A nonlinearity $\Delta$ that is static and slope-restricted on $[\alpha, \beta]$ satisfies the Zames-Falb IQC

$$
\Pi=\left[\begin{array}{cc}
-\alpha \beta\left(2-H-H^{*}\right) & \alpha(1-H)+\beta\left(1-H^{*}\right) \\
\alpha\left(1-H^{*}\right)+\beta(1-H) & -\left(2-H-H^{*}\right)
\end{array}\right]
$$

where $H(z)$ is any proper transfer function with impulse response $h:=\left(h_{0}, h_{1}, \ldots\right)$ that satisfies $\|h\|_{1} \leq 1$ and $h_{k} \geq 0$ for all $k$.

Proof. See for example [7].

Remark 15. The Zames-Falb IQC (6) admits the factorization

$$
\Psi=\left[\begin{array}{cc}
\beta(1-H) & -(1-H) \\
-\alpha & 1
\end{array}\right] \quad \text { and } \quad M=\left[\begin{array}{ll}
0 & 1 \\
1 & 0
\end{array}\right]
$$

In general, for a given fixed $\rho$, only a subset of the Zames-Falb IQCs will be $\rho$-IQCs. We now give a characterization of this subset.

Theorem 16 (Zames-Falb $\rho$-IQC). Suppose $\Delta$ is static and slope-restricted on $[\alpha, \beta]$. Then $\Delta \in \operatorname{IQC}(\Pi(z), \rho)$ where $\Pi$ is the Zames-Falb IQC (6) and H satisfies the additional constraint

$$
\sum_{k=0}^{\infty} \rho^{-2 k} h_{k} \leq 1
$$

Proof. The proof involves rewriting the IQC as a discrete-time sum which can be split into parts that can separately be shown to be nonnegative. See the Appendix for the full proof. 


\subsection{Multiple IQCs}

Much like how multiple IQCs can give more precise $L_{2}$ gain bounds, multiple $\rho$-IQCs can give more precise convergence rates. We present numerical examples with both pointwise and dynamic $\rho$-IQCs. Consider a stable discrete-time LTI system $G(z)$ in feedback with the sigmoidal nonlinearity $\Delta(x)=b \arctan (x)$. This interconnection is shown in Fig. 5 .

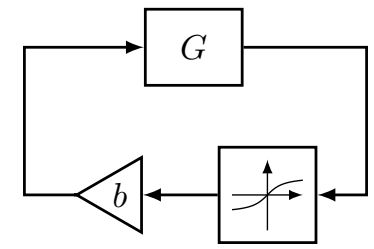

Figure 5: LTI system $G$ in feedback with the static nonlinearity $\Delta(x)=b \arctan (x)$.

Since this nonlinearity is static, in the $[0, b]$ sector, and $[0, b]$ slope-restricted, it satisfies the following $\rho$-IQCs

$$
\begin{array}{ll}
\Pi_{\mathrm{n}}(z):=\left[\begin{array}{cc}
b^{2} & 0 \\
0 & -1
\end{array}\right] & \text { (norm-bounded) } \\
\Pi_{0}(z):=\left[\begin{array}{cc}
0 & b \\
b & -2
\end{array}\right] & \text { (sector bounded) } \\
\Pi_{k}(z):=\left[\begin{array}{cc}
0 & b\left(1-\rho^{2 k} \bar{z}^{-k}\right) \\
b\left(1-\rho^{2 k} z^{-k}\right) & -2+\rho^{2 k}\left(z^{-k}+\bar{z}^{-k}\right)
\end{array}\right] & \text { (off-by- } k \text { Zames-Falb) }
\end{array}
$$

where we can choose any $k=1,2, \ldots$

A tight bound. For our first example, we analyzed the following LTI system ${ }^{1}$

$$
G_{1}(z):=-\frac{(z+1)(10 z+9)}{(2 z-1)(5 z-1)(10 z-1)}
$$

We solved the feasibility LMI (5) using MATLAB together with the CVX package [5, 6] to find the fastest guaranteed rate of convergence. We searched over positive linear combinations of subsets of the IQCs (8)-(10). Fig. 6 shows the rate bounds achieved as a function of which IQCs were used. For the particular choice $b=1$, Fig. 7 shows sample state trajectories.

The true exponential rate can be found by linearizing the system about its equilibrium point. Namely, $\Delta(x) \approx b x$. Formally, this is an application of Lyapunov's indirect method [10, Thm. 4.13]. The result is that the decay rate should correspond to the maximal pole magnitude of the closed-loop map $G(z) /(1-b G(z))$. We display the true exponential rate as the dashed black curve in Fig. 6 and Fig. 7.

For this example, the $\rho$-IQC approach yields a tight upper bound to the true exponential rate when we use a combination of the sector and off-by-1 IQCs.

A loose bound. The $\rho$-IQC approach does not always achieve tight bounds as in the previous example. Consider the same problem as before but this time using

$$
G_{2}(z):=\frac{2 z-1}{10\left(2 z^{2}-z+1\right)}
$$

\footnotetext{
${ }^{1}$ This example was inspired by the continuous time example given in [17], which showed that adding more IQCs yields better $L_{2}$ gain bounds.
} 


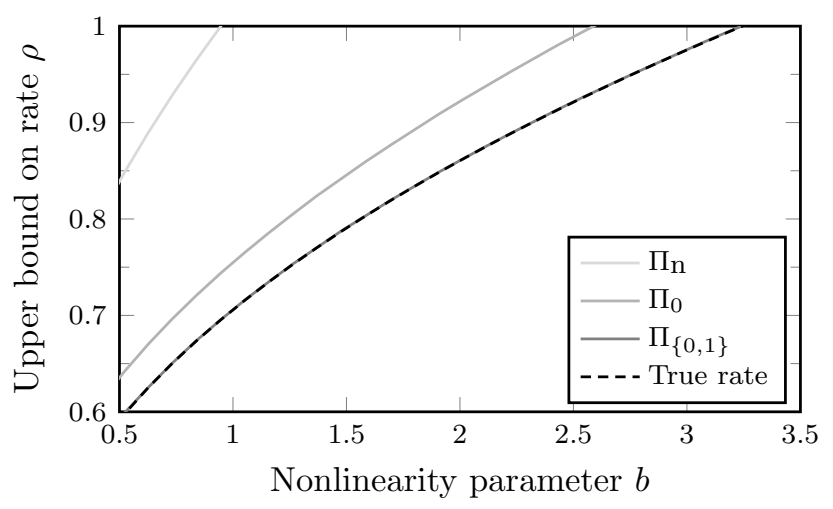

Figure 6: Upper bounds on the exponential convergence rate $\rho$ for the system $G_{1}(z)$ given in (11) in feedback as in Fig. 5. A tight bound is achieved using two $\rho$-IQCs.

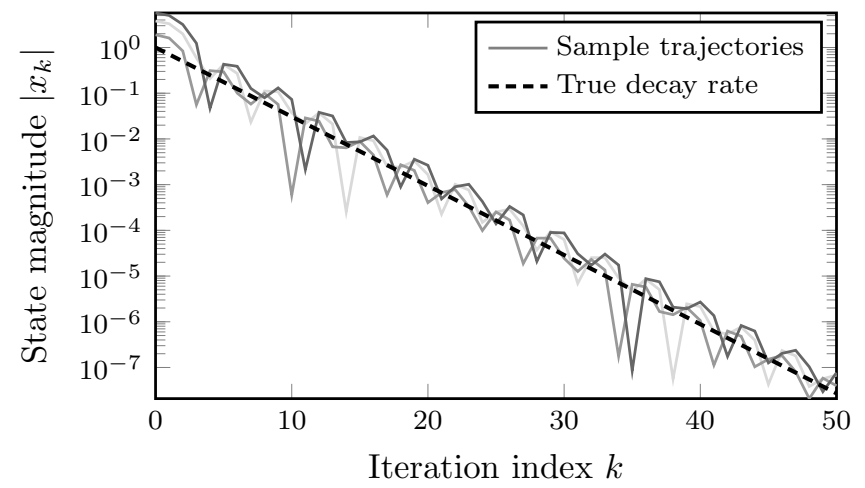

Figure 7: State decay over time of the system $G_{1}(z)$ in feedback as in Fig. 5 with $b=1$ for various initial conditions $x_{0} \in[-15,15]$. The dashed black line is $\rho^{k}$, where $\rho=.7058$ is the true rate at $b=1$ in Fig. 6 .

The rate bounds for various $\rho$-IQCs are shown in Fig. 8. This time, we again observe that using more IQCs achieves better rate bounds, but the bound is not tight even after using six IQCs.

\section{Conclusion}

We presented a modification of IQC theory that allows the certification of exponential rates. Although we only gave the $\rho$-IQC specialization for pointwise and Zames-Falb IQCs, the concept can in principle be extended to other IQCs such as, for example, uncertain time delays or slowly varying systems [13]. As the dictionary of $\rho$-IQCs is further populated, the applicability of the technique outlined herein would be correspondingly expanded.

\section{Acknowledgments}

The authors would like to thank Andrew Packard and Murat Arcak for very helpful discussions. L. Lessard is supported by AFOSR award FA9550-12-1-0339. 


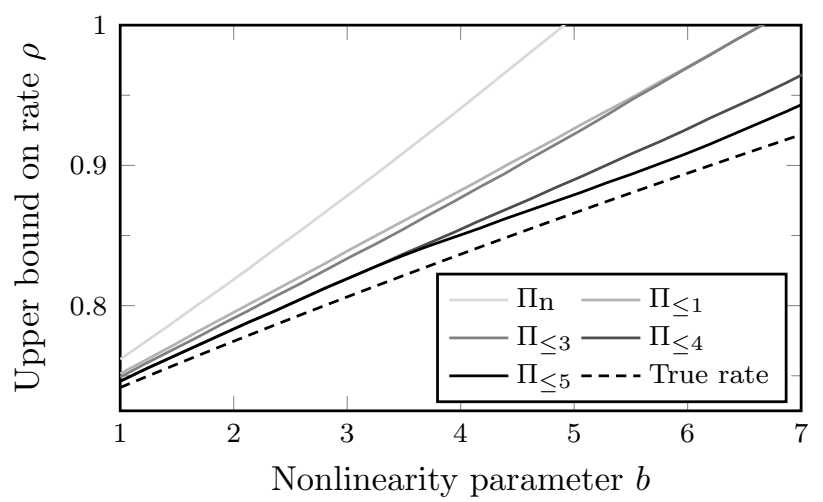

Figure 8: Upper bounds on the exponential convergence rate $\rho$ for the system $G_{2}(z)$ given in (12) in feedback as in Fig. 5. As we include more $\rho$-IQCs, we can certify tighter bounds.

\section{A Appendix}

\section{A.1 Proof of Proposition 5}

Suppose the interconnection of Fig. 3 is stable. Then there exists some $K>0$ such that for any choice of the signals $e$ and $f$ and for all $T$,

$$
\sum_{k=0}^{T}\left(\left\|w_{k}\right\|^{2}+\left\|v_{k}\right\|^{2}\right) \leq K \sum_{k=0}^{T}\left(\left\|e_{k}\right\|^{2}+\left\|f_{k}\right\|^{2}\right)
$$

The proof will follow by carefully choosing $e$ and $f$ to transform Fig. 3 into Fig. 1. To this end, note that $(A, B)$ is controllable by assumption. So there exists a finite sequence of inputs $u_{0}, \ldots, u_{n-1}$ and corresponding outputs $y_{0}, \ldots, y_{n-1}$ that drives the state of $G$ from $\xi_{0}=0$ to $\xi_{n}=x_{0}$. Therefore, if we set

$$
e_{k}=\left\{\begin{array}{ll}
\rho^{-k} u_{k} & 0 \leq k<n \\
0 & k \geq n
\end{array}, \quad f_{k}= \begin{cases}-\rho^{-k} y_{k} & 0 \leq k<n \\
0 & k \geq n\end{cases}\right.
$$

then we obtain $\xi_{n}=x_{0}$ in the interconnection of Fig. 3. Moreover, $f_{k}=e_{k}=0$ for $k \geq n$, so we may cancel the $\rho$ blocks using the fact that $\rho_{-} \circ \rho_{+}$is the identity operator. It follows that for $k \geq n$, the two interconnections become identical and therefore $\xi_{k}=x_{k-n}$.

Substituting into (13), we conclude that

$$
\sum_{k=0}^{T}\left(\left\|w_{k}\right\|^{2}+\left\|v_{k}\right\|^{2}\right) \leq K \sum_{k=0}^{n-1}\left(\left\|e_{k}\right\|^{2}+\left\|f_{k}\right\|^{2}\right)
$$

The right-hand side of (14) is independent of $T$, but (14) holds for all $T$ so we must have

$$
\lim _{k \rightarrow \infty}\left\|w_{k}\right\|=0 \quad \text { and } \quad \lim _{k \rightarrow \infty}\left\|v_{k}\right\|=0
$$

For $k \geq n$, we have $w_{k}=\rho^{-k} u_{k}$ and $v_{k}=\rho^{-k} y_{k}$. Therefore there exists some constant $c>0$ such that

$$
\left\|u_{k}\right\| \leq c \rho^{k} \quad \text { and } \quad\left\|y_{k}\right\| \leq c \rho^{k}
$$

Now $(A, C)$ is observable by assumption, so let $L$ be such that the eigenvalues of $A+L C$ are all zero. Rewrite the dynamics of $G$ as

$$
x_{k+1}=\bar{A} x_{k}+\bar{B} h_{k}
$$


where $\bar{A}:=A+L C, \bar{B}:=\left[\begin{array}{ll}L D+B & -L\end{array}\right]$, and $h_{k}:=\left[\begin{array}{ll}u_{k}^{\top} & y_{k}^{\top}\end{array}\right]^{\top}$. Iterating (15), we obtain

$$
x_{k}=\bar{A}^{k} x_{0}+\sum_{i=0}^{k-1} \bar{A}^{k-1-i} \bar{B} h_{i}
$$

Since all eigenvalues of $\bar{A}$ are zero, $\bar{A}$ is nilpotent and so $\bar{A}^{n}=0$. For $k \geq n$, (16) therefore becomes

$$
x_{k}=\sum_{i=0}^{n-1} \bar{A}^{n-1-i} \bar{B} h_{k-n+i}
$$

We can now bound the state using the triangle inequality.

$$
\begin{aligned}
\left\|x_{k}\right\| & \leq \underbrace{\left\|\left[\begin{array}{lll}
\bar{A}^{n-1} \bar{B} \quad \ldots & \bar{A} \bar{B} & \bar{B}
\end{array}\right]\right\|}_{\gamma} \sum_{i=k-n}^{k-1}\left\|h_{i}\right\| \\
& \leq \gamma c\left(\frac{\rho^{-n}-1}{1-\rho}\right) \rho^{k}
\end{aligned}
$$

and this completes the proof.

\section{A.2 Proof of Theorem 16}

We will prove this general result by first considering the simpler case where the slope restriction is on $[\alpha, \beta]=[0, \infty]$ and $H(z)=\rho^{2 j} z^{-j}$. Note that this choice trivially satisfies (7). In this case, the $\Pi$ from (6) becomes

$$
\Pi=\left[\begin{array}{cc}
0 & 1-\rho^{2 j} \bar{z}^{-j} \\
1-\rho^{2 j} z^{-j} & 0
\end{array}\right]
$$

where $\bar{z}$ denotes the complex conjugate of $z$. We call (17) the "off-by- $j$ " Zames-Falb IQC. We would like to show that $\Delta \in \operatorname{IQC}(\Pi(z), \rho)$. Appealing to Definition 6 and Remarks 10 and 15 , this amounts to proving that

$$
\sum_{k=0}^{\infty} \rho^{-2 k} u_{k}^{\top}\left(y_{k}-\rho^{2 j} y_{k-j}\right) \geq 0
$$

We will prove (18) by borrowing the approach from [12]. If $\Delta$ is multidimensional, we require that $\Delta$ be the gradient of a potential function [7]. By the assumption that $\Delta$ is slope-restricted on $[0, \infty]$, we have

$$
(\Delta(x)-\Delta(y))^{\top}(x-y) \geq 0 \text { holds for all } x, y
$$

In other words, $\Delta$ is monotone. Now define the scalar function $g$ such that $\nabla g=\Delta$. By Kachurovskii's theorem, $g$ is convex and we have

$$
g(y) \geq g(x)+\Delta(x)^{\top}(y-x) \text { for all } x, y
$$

Moreover, setting $(x, y) \mapsto\left(y_{k}, 0\right)$ or $(x, y) \mapsto\left(y_{k}, y_{k-j}\right)$ leads to the two inequalities:

$$
\begin{aligned}
u_{k}^{\top} y_{k} & \geq g\left(y_{k}\right) \\
u_{k}^{\top}\left(y_{k}-y_{k-j}\right) & \geq g\left(y_{k}\right)-g\left(y_{k-j}\right)
\end{aligned}
$$


We will assume for simplicity that $g(x) \geq 0$ for all $x$. Substituting (19) and (20) into the left-hand side of (18), the partial sum from 0 to $T$ is:

$$
\begin{aligned}
& \sum_{k=0}^{T} \rho^{-2 k} u_{k}^{\top}\left(y_{k}-\rho^{2 j} y_{k-j}\right) \\
& =\sum_{k=0}^{T} \rho^{-2 k}\left(\left(1-\rho^{2 j}\right) u_{k}^{\top} y_{k}+\rho^{2 j} u_{k}^{\top}\left(y_{k}-y_{k-j}\right)\right) \\
& \geq \sum_{k=0}^{T} \rho^{-2 k}\left(\left(1-\rho^{2 j}\right) g\left(y_{k}\right)+\rho^{2 j}\left(g\left(y_{k}\right)-g\left(y_{k-j}\right)\right)\right) \\
& =\sum_{k=0}^{T} \rho^{-2 k}\left(g\left(y_{k}\right)-\rho^{2 j} g\left(y_{k-j}\right)\right) \\
& =\sum_{k=T-j+1}^{T} \rho^{-2 k} g\left(y_{k}\right) \geq 0
\end{aligned}
$$

Since each partial sum is nonnegative, the infinite sum (which must converge) is also nonnegative, and therefore we have proven (18). For the case of general $[\alpha, \beta]$, observe that $\Pi$ from (6) may be factored as

$$
\Pi=\left[\begin{array}{cc}
\beta & -1 \\
-\alpha & 1
\end{array}\right]^{\top}\left[\begin{array}{cc}
0 & 1-H(z)^{*} \\
1-H(z) & 0
\end{array}\right]\left[\begin{array}{cc}
\beta & -1 \\
-\alpha & 1
\end{array}\right]
$$

So the above proof holds verbatim if we make the substitution $(y, u) \mapsto(\beta y-u, u-\alpha y)$.

Now we consider the case of a more general $H(z)$. Suppose $H(z)=\sum_{k=0}^{\infty} h_{k} z^{-k}$ where $h_{k}$ satisfies (7). Then,

$$
\begin{aligned}
1-H(z) & =1-\sum_{k=0}^{\infty} h_{k} z^{-k} \\
& =\underbrace{1-\sum_{k=0}^{\infty} \rho^{-2 k} h_{k}}_{c}+\sum_{k=0}^{\infty} \rho^{-2 k} h_{k}\left(1-\rho^{2 k} z^{-k}\right) \\
& =c\left(1-H_{s}\right)+\sum_{k=0}^{\infty} \rho^{-2 k} h_{k}\left(1-H_{k}(z)\right)
\end{aligned}
$$

where $H_{k}(z)=\rho^{2 k} z^{-k}$ and $H_{s}=0$. Note that $H_{k}(z)$ corresponds the off-by- $k$ Zames-Falb IQC, which we proved above is a $\rho$-IQC. Also, $H_{s}$ corresponds to the sector IQC, which is also a $\rho$-IQC. Now note that the general Zames-Falb IQC (6) is linear in $I-H$ and $I-H^{*}$. Therefore, $\Pi(z)$ is a positive linear combination of $\rho$-IQCs and must therefore be a $\rho$-IQC itself.

\section{References}

[1] R. Boczar, L. Lessard, and B. Recht. Exponential convergence bounds using integral quadratic constraints. arXiv preprint arXiv:1503.07222, 2015.

[2] S. Boyd and L. El Ghaoui. Method of centers for minimizing generalized eigenvalues. Linear algebra and its applications, 188:63-111, 1993. 
[3] S. P. Boyd, L. El Ghaoui, E. Feron, and V. Balakrishnan. Linear matrix inequalities in system and control theory, volume 15. SIAM, 1994.

[4] M. Corless and G. Leitmann. Bounded controllers for robust exponential convergence. Journal of Optimization Theory and Applications, 76(1):1-12, 1993.

[5] M. Grant and S. Boyd. Graph implementations for nonsmooth convex programs. In V. Blondel, S. Boyd, and H. Kimura, editors, Recent Advances in Learning and Control, Lecture Notes in Control and Information Sciences, pages 95-110. SpringerVerlag Limited, 2008. http://stanford.edu/ boyd/graph_dcp.html.

[6] M. Grant and S. Boyd. CVX: Matlab software for disciplined convex programming, version 2.1. http://cvxr.com/cvx, 2014.

[7] W. P. Heath and A. G. Wills. Zames-Falb multipliers for quadratic programming. In IEEE Conference on Decision and Control, pages 963-968, 2005.

[8] J. Jerez, P. Goulart, S. Richter, G. Constantinides, E. Kerrigan, and M. Morari. Embedded online optimization for model predictive control at megahertz rates. IEEE Transactions on Automatic Control, 59(12):3238-3251, 2014.

[9] U. Jönsson. A nonlinear Popov criterion. In IEEE Conference on Decision and Control, volume 4, pages 3523-3527, 1997.

[10] H. K. Khalil. Nonlinear systems (3rd edition). Prentice Hall, 2002.

[11] K. Konishi and H. Kokame. Robust stability of Lure systems with time-varying uncertainties: A linear matrix inequality approach. International Journal of Systems Science, 30(1):3-9, 1999.

[12] L. Lessard, B. Recht, and A. Packard. Analysis and design of optimization algorithms via integral quadratic constraints. arXiv preprint arXiv:1408.3595, 2014.

[13] A. Megretski and A. Rantzer. System analysis via integral quadratic constraints. IEEE Transactions on Automatic Control, 42(6):819-830, 1997.

[14] A. Rantzer. On the Kalman-Yakubovich-Popov lemma. Systems $\&$ Control Letters, 28(1):7-10, 1996.

[15] A. Rantzer and A. Megretski. System analysis via Integral Quadratic Constraints, part II. Technical Report ISRN LUTFD2/TFRT--7559--SE, Department of Automatic Control, Lund University, Sweden, 1997.

[16] M. G. Safonov and V. V. Kulkarni. Zames-Falb multipliers for MIMO nonlinearities. In American Control Conference, volume 6, pages 4144-4148, 2000.

[17] C. Scherer. Lecture notes for the course "linear matrix inequalities in control". http://www.dcsc.tudelft.nl/ cscherer/lmi/lec5.pdf. Accessed on March 22, 2015.

[18] P. Seiler. Stability analysis with dissipation inequalities and integral quadratic constraints. IEEE Transactions on Automatic Control, 60(6):1704-1709, 2015.

[19] J. C. Willems. Dissipative dynamical systems part I: General theory. Archive for Rational Mechanics and Analysis, 45(5):321-351, 1972.

[20] G. Zames. On the input-output stability of time-varying nonlinear feedback systems - Part I: Conditions derived using concepts of loop gain, conicity, and positivity, and Part II: Conditions involving circles in the frequency plane and sector nonlinearities. IEEE Transactions on Automatic Control, 11(2,3):228-238,465-476, 1966.

[21] G. Zames and P. Falb. Stability Conditions for Systems with Monotone and SlopeRestricted Nonlinearities. SIAM Journal on Control, 6(1):89-108, 1968. 